Notfall Rettungsmed 2016 $\cdot 19: 531-532$

DOI 10.1007/s10049-016-0221-2

Online publiziert: 6. September 2016

๑) Springer-Verlag Berlin Heidelberg 2016

CrossMark

\section{B. Gliwitzky ${ }^{1}$ C. Wrede ${ }^{2}$}

${ }^{1}$ MegaMed GbR, Annweiler, Deutschland

${ }^{2}$ Interdisziplinäres Notfallzentrum, Berlin, Deutschland

\title{
Weiterbildung in der Notfallmedizin
}

Sehr geehrte Leserinnen und Leser,

die aktuelle Ausgabe der Notfall + Rettungsmedizin beschäftigt sich mit einem seit Jahren aktuellen und vielfach kontrovers diskutiertem Thema: der Aus-, Fort- und Weiterbildung auf dem gesamten Gebiet der Notfallmedizin. Wurde noch vor 20 Jahren Notfallmedizin nahezu ausschließlich präklinisch definiert, umfasst das heutige System deutlich mehr als die prähospitale Versorgung von kritisch kranken Patienten. Gleichzeitig ist der Anspruch an die prä- und innerklinische Notfallmedizin heute deutlich höher als noch vor 20 Jahren, was erheblich steigende Anforderungen sowohl an die Ausbildung, aber auch die kontinuierliche Fort- und Weiterbildung von Rettungsfachpersonal, Ärzten und Pflegekräften stellt. Dass hierbei aktuell Defizite bestehen, wird auch in den Leitlinien des European Resuscitation Council 2010 und 2015 deutlich, in denen es heißt: „Einige Studien zeigen, dass Ärzte und Pflegepersonal mangelhaftes Wissen und geringe Fertigkeiten in der Akutbehandlung aufweisen, z. B. in der Sauerstofftherapie, der Flüssigkeits- und Elektrolytbalance, der Schmerzbekämpfung, des Einverständnisses von Patienten, der Pulsoxymetrie und der Dosierung von Medikamenten. Aus- und Fortbildung des medizinischen Personals sind ein wesentlicher Teil der Einführung eines Systems zur Vorbeugung von Kreislaufstillständen [1].“ In den Beiträgen dieses Leitthemenhefts werden Konzepte vorgestellt, um die Qualifikation der verschiedenen, an der Notfallversorgung beteiligten Berufsgruppen zu verbessern.
Wir haben dies in einem Heft in der Überzeugung zusammengefasst, dass sowohl gute Kenntnisse als auch ein gutes Zusammenspiel aller beteiligten Akteure letztlich für den Behandlungserfolg entscheidend sind.

Zentrale Notaufnahmen und eine intensive Diskussion um die richtigen Qualifikationen für das eingesetzte ärztliche und pflegerische Personal beschäftigen uns seit einigen Jahren und haben zu unterschiedlichen Empfehlungen geführt $[2,3]$.

\section{》) Die notwendige Qualifikation von Ärzten in den Notaufnahmen wurde sehr kontrovers diskutiert}

Die notwendige ärztliche Qualifikation von Ärzten in den Notaufnahmen wurde sehr kontrovers diskutiert und reichte in der Bandbreite von einer Ablehnung von jeglichen speziellen Qualifikationen bis hin zu einem Facharzt für Notfallmedizin. 2014 wurde von der Ärztekammer Berlin eine dreijährige Zusatzweiterbildung ,Klinische Notfall- und Akutmedizin" eingerichtet, und aktuell gibt es eine Einigung der Fachgesellschaften auf ein Konzept für eine Zusatzweiterbildung, die zu einem gemeinsamen Vorschlag von DGINA und DIVI bei der Bundesärztekammer führen soll [4]. Der aktuelle Stand der Diskussion wird von Wyrwich und Gries vorgestellt. In der Qualifikation der Notfallpflege gibt es ebenfalls in den letzten Jahren mehrere neue Entwicklungen. Es wurde ein DGINA Curriculum zur Notfallpflege veröffentlicht und einige Bundesländer haben eine staatli- che Fachweiterbildung Notfallpflege eingerichtet bzw. sind auf dem Weg dorthin. Dies wird durch Wedler et al. dargestellt. Jenseits der Weiterbildung ist die kontinuierliche berufsgruppenübergreifende Fortbildung in den Notaufnahmen für eine gute Patientenversorgung wichtig, und Simulationstrainings haben sich auch in diesem Rahmen als sinnvoll und notwendig erwiesen.

Innerklinische Notfälle spielen eine immer größere Rolle in den Diskussionen, $70 \%$ der innerklinischen Reanimationen lassen sich wohlmöglich durch geeignete Frühwarnsysteme (Early Warning Scores) und den frühen Einsatz von medizinischen Notfallteams (MET) vermeiden [1]. Entsprechende Schulungen des Personals sind hier unabdingbar.

Auch in der präklinischen Versorgung haben sich die Anforderungen an die diagnostischen und therapeutischen Kompetenzen beim Rettungsfachpersonal und den Notärzten deutlich erhöht. Beispiele für größere technische und medizintechnische Möglichkeiten im Rettungsdienst sind die Telemedizin, die flächendeckende Einführung des präklinischen 12-Kanal-EKG oder auch die nichtinvasive Beatmung. Die zielgerichtete Zuweisung von Notfallpatienten in die für den Patienten geeignete Klinik wird aufgrund einer zunehmenden Spezialisierung der Kliniken immer bedeutsamer, um zeitkritische Krankheitsbilder in einer notwendigen Zeitspanne versorgen zu können. Ein großer Schritt in die richtige Richtung war sicherlich die Verabschiedung des Notfallsanitätergesetzes [5]. In den nächsten Jahren wird dies zu einer deutlichen Verbesserung der Qua- 
lität bei der präklinischen Versorgung beitragen können. Auch die Anforderungen an die Aus-und Fortbildung der Notärzte müssen zukünftig deutlich steigen. Marung et al. und Reifferscheid et al. gehen in ihren Leithemenbeiträgen diesen Herausforderungen nach und zeigen mögliche Lösungen auf. Dabei spielen nicht nur fachliche Aspekte eine Rolle, sondern zunehmend auch Kompetenzen in der Zusammenarbeit der verschiedenen Professionen und an den Nahtstellen der unterschiedlichen Arbeitsbereiche. Schmitz et al. beschreiben in ihrem Beitrag eine mögliche Umsetzung in Freiburg. Das Feld der Hilfeleistungsersuchenden ist breiter geworden, beispielsweise haben es der gleiche Notfallsanitäter und der gleiche Notarzt in den Einsätzen sowohl mit Versorgungsproblemen allein lebender Senioren als auch mit Reanimationen oder mit einem schweren Trauma eines Kindes zu tun. Verschiedene Einsätze mit unterschiedlichsten Anforderungen erfordern unterschiedliche Kompetenzen, und Routine bei seltenen Einsätzen kann gar nicht entstehen. Auf der anderen Seite sind dies häufig Erkrankungen oder Verletzungen, die ein umgehendes Handeln auf höchstem Niveau erfordern.

$\mathrm{Zu}$ einer Notfallmedizin auf hohem Niveau in Präklinik und Klinik gehört eine qualitativ hochwertige Fort- und Weiterbildung, die damit aktiv zur Patientensicherheit beiträgt. Geeignete Konzepte zur zielgerichteten Fortbildung sind zu definieren und auch in ihrer Effizienz $\mathrm{zu}$ messen. Eine qualitativ hochwertige Umsetzung ist jedoch mit Zeit und Kosten verbunden, und die Kostenträger müssen bereit sein, einen Anteil der Arbeitszeit als Fortbildungszeit festzulegen und auch zu bezahlen. Rettungsdienst, Notaufnahmen und Intensivstationen sind Hochsicherheitsarbeitsplätze. Sie sind mit dem Arbeitsplatz im Cockpit eines Verkehrsflugzeugs und in einem Atomkraftwerk vergleichbar. Stellen Sie sich vor, Piloten würden nicht regelmäBig im Simulator gemeinsam trainieren! Undenkbar! Fangen wir an umzudenken. Gut qualifiziertes Personal kostet Geld. Dieses Geld scheint im Zuge zunehmend fehlender Fachkräfte gut investiert, um weiterhin mit der medizintechnischen
Entwicklung und den therapeutischen Optionen auf breiter Basis Schritt zu halten.

\section{Herzlichst}

Ihre

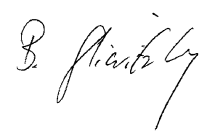

Bernhard Gliwitzky

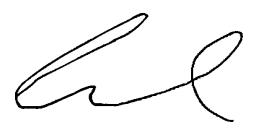

Prof. Christian Wrede

\section{Korrespondenzadresse}

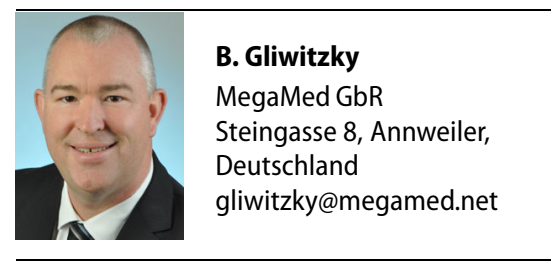

Interessenkonflikt. B. Gliwitzky erklärt, dass er Geschäftsführer von MegaMed Notfallmanagement GbR und Geschäftsführer der GRC Akademie GmbH ist. C. Wrede gibt an, dass kein Interessenkonflikt besteht.

\section{Literatur}

1. Soar J, Nolan JP, Böttiger BW et al (2015) European Resuscitation Council Guidelines for Resuscitation 2015 Section 3. Adult advanced life support. Resuscitation 95:100-147

2. Fleischmann T, Walter B (2007) Interdisziplinäre Notaufnahmen in Deutschland - Eine Anlaufstelle für alle Notfälle. Dtsch Arztebl 104(46):A3164

3. Zur Problematik zentraler Notaufnahmen Gemeinsame Stellungnahme der Deutschen Gesellschaft für Chirurgie (DGCH) und der Deutschen Gesellschaft für Innere Medizin (DGIM). http://www.dgim.de/portals/pdf/sn_zentrale_ notaufnahme_dgch_dgim.pdf. Zugegriffen: Juni 2016

4. Gerst T (2016) Notfallmedizin:Verständigung über Zusatzweiterbildung. Dtsch Arztebl 113(15):A694/B-586/C-578

5. Das Notfallsanitätergesetz. https://www.gesetzeim-internet.de/notsang/BJNR134810013.html. Zugegriffen:22. Juni 2016
Ihre Vorteile wenn Sie in der Notfall+Rettungsmedizin publizieren:

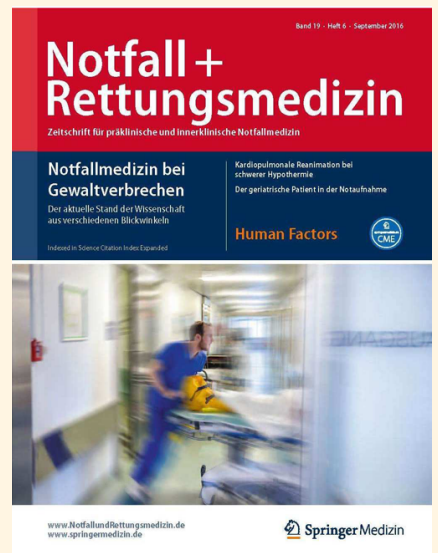

Mit der Publikation Ihrer Fallberichte, Originalarbeiten oder Übersichten in der Notfall+Rettungsmedizin

- erreichen Sie auf einen Schlag knapp $\mathbf{9 0 0 0}$ Kollegen aus dem gesamten Notfallteam,

- ist Ihr Beitrag, nach dem er akzeptiert wurde, innerhalb weniger Wochen direkt online verfügbar,

- haben Sie im Peer Review-Verfahren die Möglichkeit zum fachlichen Austausch mit anderen Experten,

- bekommen Sie bei Originalien Unterstützung durch ein statistisches Fachgutachten,

- können Sie Ihre Arbeit, bei Wunsch, von Medizinrechtlern gegenlesen lassen,

- wird Ihr Beitrag vom Verlag professionell lektoriert und gesetzt.

Informationen zur Manuskripterstellung finden Sie unter "Hinweise für Autoren" auf www.notfallundrettungsmedizin.de

Bitte reichen Sie Ihren Beitrag über das online Begutachtungssystem Editorial Manager ein:

www.editorialmanager.com/nore

Während des gesamten Prozesses steht Ihnen die Redaktion als Ansprechpartner zur Verfügung:

Dr. Ines Wolff, ines.wolff@springer.com 